\title{
ADAPTIVE SCHEME FOR ACCURATE ORBIT PROPAGATION
}

\author{
Giulio Baù \\ PhD Candidate, University of Padova, CISAS "G. Colombo", via Venezia 15, 35131 Padova, Italy, \\ +390498276798, giulio.bau@unipd.it
}

\section{Claudio Bombardelli}

Research Fellow, Technical University of Madrid (UPM), ETSI Aeronauticos, Plaza Cardenal Cisneros 3, 28040 Madrid, Spain, +34 91336 3939, claudio.bombardelli@upm.es

\section{Jesús Peláez}

Professor, Technical University of Madrid (UPM), ETSI Aeronauticos, Plaza Cardenal Cisneros 3, 28040 Madrid, Spain, +3491336 6306, j.pelaez@upm.es

\begin{abstract}
Two extensions of the fast and accurate special perturbation method recently developed by Peláez et al. are presented for elliptic motion. A comparison with Peláez's method and with the very efficient Stiefel-Scheifele's method, for the problems of oblate Earth plus Moon and continuous radial thrust, shows that the new formulations can appreciably improve the accuracy of Peláez's method and have a better performance of Stiefel-Scheifele's method. Future work will be to include the two new formulations and the original one due to Peláez into an adaptive scheme for highly accurate orbit propagation.
\end{abstract}

Keywords: Orbit propagation, Variation of parameters, Two-body regularization

\section{Introduction}

Equations of motion derived by the variation of parameters (VOP) method are effective in dealing with the longterm study of the motion of asteroids, comets, as well as natural and artificial satellites subject to relatively small perturbation forces, mainly because, unlike the methods formulated in rectangular coordinates, describe the evolution of elements (or integrals of the motion), which exhibit no error propagation with respect to the unperturbed two-body problem. The VOP can be further improved introducing a two-body regularization which cancels out the dependence on the eccentricity of the propagation error of the physical time in the pure Keplerian motion. The Stiefel-Scheifele's method (Stiefel and Scheifele, 1971), which is based on the set of regular elements attached to the parametric coordinates of the well-known Kustaaheimo-Stiefel (K-S) regularization, is very accurate, or equivalently fast, especially in case of weak perturbations and long-term integrations. In addition, as noted by Arakida and Fukushima (2001), the linear character of the error growth, which characterizes the body position and the physical time, is in this case independent on the integrator used.

Recently, the performance of the Stiefel-Scheifele's method has been further improved by Fukushima (see Fukushima (2004), Arakida and Fukushima (2001) and references therein) using different techniques, in particular single and quadrupole scaling methods and VOP method, applied to the K-S regularization.

A different strategy towards the improvement of a VOP method has been followed by Peláez et al. In a recent article (Peláez et al., 2007) Peláez has proposed a new formulation for the two body-problem equations of motion, borrowing elements of rigid-body dynamics. The idea is to track the evolution of an orbital frame moving with the particle and link a new set of generalized orbital elements to this frame. The result is an improvement in accuracy (or, equivalently, computational speed) with respect to other efficient methods in orbital dynamics, such as K-S and Sperling-Burdet, and a more compact and simple formulation of the equations of motion. Another key advantage of Peláez's method is that it allows to a unique formulation for elliptic, parabolic and hyperbolic motion so that transitions from different kinds of orbits can be managed without stopping the integration. This fact is related to the choice of the fictitious time, which coincides with the true anomaly in the pure Keplerian motion. However, the consequence of this choice is that full regularization cannot be achieved due to the structure of the Sundmann transformation employed (Stiefel and Scheifele, 1971). From a practical point of view, when the orbital motion approaches a rectilinear motion, i.e. along highly eccentric orbits, the growth of the propagation error is amplified.

Two sets of regular elements are developed in the framework of Peláez's method (Peláez et al., 2007) for propagating the elliptic and hyperbolic motions respectively. The starting point of our procedure for constructing the two element formulations is the introduction of a pseudo-eccentric anomaly for the ellipse and a pseudo-hyperbolic 
anomaly for the hyperbola. We show that, for the elliptic motion, the new method improves the accuracy of Pelaez's method, and its performance is comparable to and better than Stiefel-Scheifele's method. The formulation for the hyperbolic motion will be ready very soon. Peláez's method and its two extensions will be part of an adaptive scheme of orbital propagation, which will be able to switch between the three formulations for accurately propagating the motion of a particle even in the critic situation of near-rectilinear motion. Optimal switch conditions will be found in order to meet accuracy requirements for the cases of highly eccentric orbit and planetary capture.

\section{Peláez's special perturbation method}

The starting idea of Peláez's special perturbation method (see Peláez et al. (2007)) is the decomposition of the position vector of a point mass $\mathrm{x}$ into the product of its magnitude $R=\|\mathbf{x}\|$ and its direction $\mathbf{i}=\mathbf{x} / R$. The decomposition in the projective coordinates $(R, \mathbf{i})$, so-called after Ferrándiz, is a preliminary operation in obtaining a set of linearized equations of motion (Deprit et al., 1994).

Non-dimesionalization is performed and the differential equations with respect to the non-dimensional time $\tau$ are derived for:

1. $z=1 / r$, where $r$ is the non-dimensional orbital radius;

2. $u=v_{r}$, where $v_{r}$ is the non-dimensional radial velocity;

3. $\psi=h$, where $h$ is the non-dimensional specific angular momentum;

4. the components of the unit quaternion $p=\left(p_{1}, p_{2}, p_{3}, p_{4}\right)$ related to the orbital frame $\mathcal{R}=\langle\mathbf{i}, \mathbf{j}, \mathbf{k}\rangle$. The unit vectors of $\mathcal{R}$ are defined by the relations:

$$
\mathbf{i}=\frac{\mathbf{x}}{R}, \quad \mathbf{k}=\frac{\mathbf{x} \wedge \dot{\mathbf{x}}}{\|\mathbf{x} \wedge \dot{\mathbf{x}}\|}, \quad \mathbf{j}=\mathbf{k} \wedge \mathbf{i}
$$

where $\dot{\mathbf{x}}$ is the velocity vector.

The next step done in Peláez et al. (2007) is to change the independent variable from the physical time $\tau$ to the fictitious time $\sigma$, according to the transformation:

$$
\frac{\mathrm{d} \tau}{\mathrm{d} \sigma}=\frac{r^{2}}{h}
$$

Finally, the variation of parameters technique is applied and the differential equations of the elements attached to the quantities $z, u, \psi$ and $p_{1}, p_{2}, p_{3}$ and $p_{4}$ are determined. In the next section we explain the meaning of the elements introduced by the method.

\subsection{Integrals of the motion}

One of the elements is the specific angular momentum $\psi$ and needs no explanation.

Let $\mathbf{f}_{p}=\left(f_{p x}, f_{p y}, f_{p z}\right)^{\mathrm{T}}$ be the perturbing acceleration vector (non-dimensional) expressed in the orbital frame $\mathcal{R}$, which was defined in (1). The angular velocity (non-dimensional) of $\mathcal{R}$ is given by:

$$
\boldsymbol{\omega}=\frac{r}{h} f_{p z} \mathbf{i}+\frac{h}{r^{2}} \mathbf{k}
$$

Let us consider a reference frame $\mathcal{U}$ which is rotating with respect to $\mathcal{R}$ at the angular velocity:

$$
\mathbf{\Omega}_{r e l}=-\frac{h}{r^{2}} \mathbf{k}
$$

then, the angular velocity of $\mathcal{U}$ is:

$$
\boldsymbol{\Omega}=\frac{r}{h} f_{p z} \mathbf{i}
$$

We note that when $f_{p z}$ is equal to zero the unit vectors of $\mathcal{U}$ remain fixed with respect to the inertial space. More precisely, $\mathcal{U}$ represents a family of reference frames, which are rotated with respect to $\mathcal{R}$ of $\sigma+C$ clockwise around the direction of the osculating angular momentum vector, where $C$ is an arbitrary constant. 


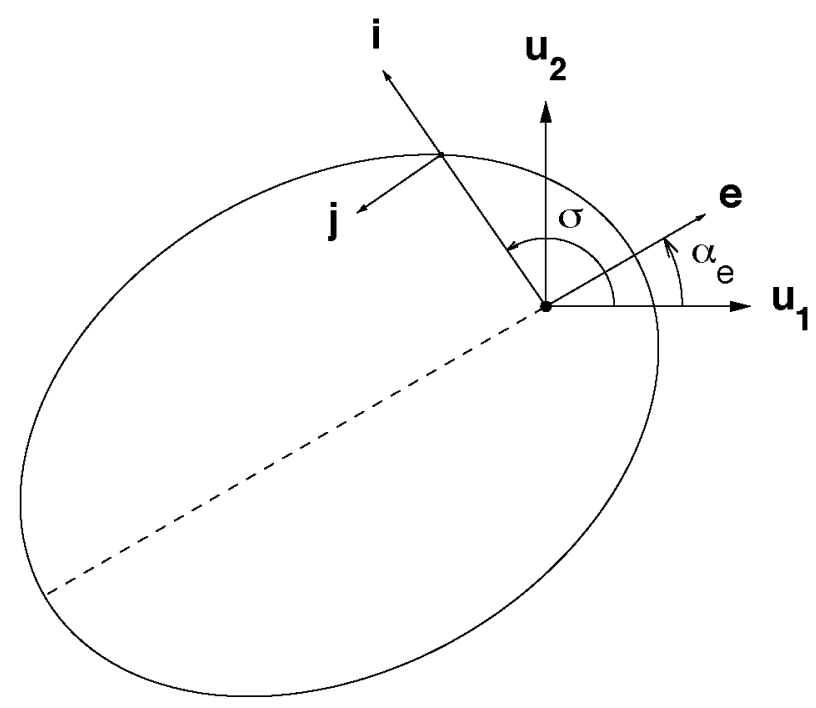

Figure 1. Orbital frames $\mathcal{R}$ and $\mathcal{U}_{0}$ (k-axis view)

For $C=0$ the corresponding orbital frame $\mathcal{U}_{0}=\left\langle\mathbf{u}_{1}, \mathbf{u}_{2}, \mathbf{u}_{3}\right\rangle$ is defined as follows:

$$
\left[\mathbf{u}_{1}, \mathbf{u}_{2}, \mathbf{u}_{3}\right]=[\mathbf{i}, \mathbf{j}, \mathbf{k}] Q,
$$

where

$$
Q=\left(\begin{array}{ccc}
\cos \sigma & \sin \sigma & 0 \\
-\sin \sigma & \cos \sigma & 0 \\
0 & 0 & 1
\end{array}\right)
$$

The unit vectors $\mathbf{u}_{1}$ and $\mathbf{u}_{2}$ are depicted in Fig. (1).

The elements attached to the quantities $z$ and $u$, named $A$ and $B$ in Peláez et al. (2007), are multiplied by $\psi$ and the new elements:

$$
q_{1}=\psi A \quad q_{2}=\psi B
$$

are introduced. Let us project the eccentricity vector on $\mathcal{R}$ :

$$
\mathrm{e}=-\frac{\mathbf{x}}{r}-\frac{1}{\mu} \mathbf{h} \wedge \dot{\mathbf{x}}=-\mathbf{i}-\frac{1}{q_{3}} \mathbf{k} \wedge(u \dot{\mathbf{i}}+s \mathbf{j}),
$$

and by exploiting the relations:

$$
\begin{aligned}
& s=q_{3}+q_{1} \cos \sigma+q_{2} \sin \sigma \\
& u=q_{1} \sin \sigma-q_{2} \cos \sigma,
\end{aligned}
$$

for, respectively, the non-dimensional transverse and radial velocity, and the identities $\mathbf{k} \wedge \mathbf{i}=\mathbf{j}$ and $\mathbf{k} \wedge \mathbf{j}=-\mathbf{i}$, Eq. (5) yields:

$$
\mathbf{e}=\left(\frac{q_{1}}{q_{3}} \cos \sigma+\frac{q_{2}}{q_{3}} \sin \sigma\right) \mathbf{i}-\left(\frac{q_{1}}{q_{3}} \sin \sigma-\frac{q_{2}}{q_{3}} \cos \sigma\right) \mathbf{j} .
$$

Equation (4) is used to project e on $\mathcal{U}_{0}$, it results:

$$
\mathbf{e}=\frac{q_{1}}{q_{3}} \mathbf{u}_{1}+\frac{q_{2}}{q_{3}} \mathbf{u}_{2},
$$

and if the new variables:

$$
\zeta_{1}=\frac{q_{1}}{q_{3}}, \quad \zeta_{2}=\frac{q_{2}}{q_{3}}
$$

are introduced into Eq. (8), we finally get:

$$
\mathbf{e}=\zeta_{1} \mathbf{u}_{1}+\zeta_{2} \mathbf{u}_{2} .
$$


The previous equation indicates that $\zeta_{1}$ and $\zeta_{2}$ are the projections of the eccentricity vector on the unit vectors $\mathbf{u}_{1}$ and $\mathbf{u}_{2}$ :

$$
\begin{aligned}
& \zeta_{1}=e \cos \alpha_{e} \\
& \zeta_{2}=e \sin \alpha_{e},
\end{aligned}
$$

where $\alpha_{e}$, which is shown in Fig. (1), is the angle between $\mathbf{u}_{1}$ and e. At the initial time $t_{0}, \sigma$ is equal to the arbitrary constant $\sigma_{0}$. By setting $\sigma_{0}$ equal to the initial true anomaly $\vartheta\left(t_{0}\right)$, it follows $\alpha_{e}\left(t_{0}\right)=0$, which means that $\mathbf{u}_{1}$ is parallel to the eccentricity vector at time $t_{0}$.

We deal now with the elements attached to the unit quaternion $p$. They represent the components of the unit quaternion related to the orbital frame $\mathcal{R}_{0}=\left\langle\mathbf{i}_{0}, \mathbf{j}_{0}, \mathbf{k}_{0}\right\rangle$, whose unit vectors are defined by:

$$
\left[\mathbf{i}_{0}, \mathbf{j}_{0}, \mathbf{k}_{0}\right]=[\mathbf{i}, \mathbf{j}, \mathbf{k}] Q
$$

where

$$
Q=\left(\begin{array}{ccc}
\cos \triangle \sigma & \sin \triangle \sigma & 0 \\
-\sin \triangle \sigma & \cos \triangle \sigma & 0 \\
0 & 0 & 1
\end{array}\right)
$$

with $\triangle \sigma=\sigma-\sigma_{0}$. We recognize that $\mathcal{R}_{0}$ belongs to the family of frames indicated by $\mathcal{U}$, and, in particular, it corresponds to the choice $C=-\sigma_{0}$. As a consequence, this frame is invariant when the motion is unperturbed, and also when the disturbing acceleration is locked within the orbital plane. According to our assumption on $\sigma_{0}$, the frame $\mathcal{R}_{0}$ is permanently rotated of $\vartheta\left(t_{0}\right)$ with respect to $\mathcal{U}_{0}$.

In general, for each choice of the value of $C$, it exists a $S O(3)$ rotation with respect to an inertial reference frame based on the following Euler angles: the right ascension of the ascending node $\Omega$, the inclination $i$ and the angle:

$$
\bar{\omega}=\omega-\alpha_{e}-C,
$$

where $\omega$ is the argument of periapsis. For example, we have $\bar{\omega}=\omega-\alpha_{e}+\sigma_{0}$ for the reference frame $\mathcal{R}_{0}$, and $\bar{\omega}=\omega-\alpha_{e}$ for $\mathcal{U}_{0}$. The angles $\Omega, i$ and $\bar{\omega}$ are integrals of the motion, even when the acceleration vector is not zero and lies on the orbital plane.

Once the orbital frames $\mathcal{U}_{0}$ and $\mathcal{R}_{0}$ are defined, the following elements can be introduced:

$$
\boldsymbol{\alpha}=\left(\begin{array}{lllllll}
\zeta_{1} & \zeta_{2} & \zeta_{3} & p_{10} & p_{20} & p_{30} & p_{40}
\end{array}\right)^{\mathrm{T}}
$$

where $\zeta_{1}$ and $\zeta_{2}$ are the projections of the eccentricity vector on $\mathbf{u}_{1}$ and $\mathbf{u}_{2}$ respectively, $\zeta_{3}$ is the inverse of the non-dimensional specific angular momentum, and $p_{10}, p_{20}, p_{30}$ and $p_{40}$ are the components of the unit quaternion which defines the orientation of $\mathcal{R}_{0}$. The differential equations of these elements take the form:

$$
\begin{aligned}
& \frac{\mathrm{d} \zeta_{1}}{\mathrm{~d} \sigma}=2 g\left\{\hat{s} \sin \sigma f_{p x}+\left[\zeta_{1}+(1+\hat{s}) \cos \sigma\right] f_{p y}\right\} \\
& \frac{\mathrm{d} \zeta_{2}}{\mathrm{~d} \sigma}=2 g\left\{-\hat{s} \cos \sigma f_{p x}+\left[\zeta_{2}+(1+\hat{s}) \sin \sigma\right] f_{p y}\right\} \\
& \frac{\mathrm{d} \zeta_{3}}{\mathrm{~d} \sigma}=-2 g \zeta_{3} f_{p y} \\
& \frac{\mathrm{d} p_{10}}{\mathrm{~d} \sigma}=g f_{p z}\left[\cos \left(\sigma-\sigma_{0}\right) p_{40}-\sin \left(\sigma-\sigma_{0}\right) p_{30}\right] \\
& \frac{\mathrm{d} p_{20}}{\mathrm{~d} \sigma}=g f_{p z}\left[\cos \left(\sigma-\sigma_{0}\right) p_{30}+\sin \left(\sigma-\sigma_{0}\right) p_{40}\right] \\
& \frac{\mathrm{d} p_{30}}{\mathrm{~d} \sigma}=-g f_{p z}\left[\cos \left(\sigma-\sigma_{0}\right) p_{20}-\sin \left(\sigma-\sigma_{0}\right) p_{10}\right] \\
& \frac{\mathrm{d} p_{40}}{\mathrm{~d} \sigma}=-g f_{p z}\left[\cos \left(\sigma-\sigma_{0}\right) p_{10}+\sin \left(\sigma-\sigma_{0}\right) p_{20}\right]
\end{aligned}
$$


where:

$$
\begin{aligned}
& \hat{s}=1+\zeta_{1} \cos \sigma+\zeta_{2} \sin \sigma \\
& g=\frac{1}{2 \zeta_{3}^{4} \hat{s}^{3}} .
\end{aligned}
$$

Finally, we consider the differential equation of time:

$$
\frac{\mathrm{d} \tau}{\mathrm{d} \sigma}=\frac{1}{\zeta_{3}^{3} \hat{s}^{2}} .
$$

\subsection{Singularities of Peláez's method}

Note that $g$, which appears on the right-hand sides of Eqs. (10) - (16), can also be written in the form:

$$
g=\frac{h^{4}}{2(1+e \cos \vartheta)^{3}},
$$

where $h$ is the non-dimensional specific angular momentum, $e$ is the eccentricity and $\vartheta$ is the true anomaly. Therefore, the differential equations of the elements become singular when the term $1+e \cos \vartheta$ is zero. This situation occurs when:

1. $e=1$ and $\vartheta=\pi$ : rectilinear ellipse at apoapsis and parabola for $r$ equal to infinity;

2. $\cos \vartheta=-1 / e$ : hyperbola for $r$ equal to infinity.

From a practical point of view there are two cases in which the accuracy of the method might be deteriorated: highly eccentric orbits near the apoapsis, and hyperbolic orbits near the asymptotes.

As regard the time equation, which is Eq. (2), it contains a singularity for $h$ equal to zero, i.e. when the motion is rectilinear.

\section{Elliptic motion}

For the time being we deal with elliptic motion. We will present in a separate paper an analogous formulation for hyperbolic motion.

\subsection{First three elements}

We express the perturbed two-body problem equation in the orbital frame $\mathcal{R}$, which is defined by relations (1), and project on the i-axis. If non-dimensional quantities are employed (see Peláez et al. (2007)), the resulting equation takes the form:

$$
\frac{\mathrm{d}^{2} r}{\mathrm{~d} \tau^{2}}-\frac{h^{2}}{r^{3}}+\frac{1}{r^{2}}=\mathbf{f}_{p} \cdot \mathbf{i}
$$

It is a known fact (see Boccaletti and Pucacco (1999)) that the eccentric anomaly is a regularizing variable for Kepler motion, and for this reason in Eq. (19) we change the independent variable from the non-dimensional time $\tau$ to the fictitious time $\mathcal{E}$ through the transformation:

$$
\frac{\mathrm{d} \tau}{\mathrm{d} \mathcal{E}}=r \sqrt{a}
$$

where $a$ is the non-dimensional semi-major axis. Let us introduce the eccentric anomaly $E$ and consider the time derivative of $E$ (see Battin (1999), p. 503), which is given by:

$$
\frac{\mathrm{d} E}{\mathrm{~d} \tau}=\frac{\mathrm{d} \mathcal{E}}{\mathrm{d} \tau}+\frac{\partial E}{\partial \mathbf{v}} \cdot \mathbf{f}_{p},
$$

where $\mathbf{v}$ is the non-dimensional velocity vector. If we impose $\mathbf{f}_{p}=\mathbf{0}$ for $t \geq t^{*} \geq 0$, being $t^{*}$ an arbitrary instant of time, we see that $\mathcal{E}$ will differ from $E$ by a constant term. 
For a generic quantity $x$ the two relations below are derived:

$$
\begin{aligned}
& \frac{\mathrm{d} x}{\mathrm{~d} \tau}=\frac{1}{r \sqrt{a}} \frac{d x}{d \mathcal{E}} \\
& \frac{\mathrm{d}^{2} x}{\mathrm{~d} \tau^{2}}=\frac{1}{r^{2} a}\left[\frac{\mathrm{d}^{2} x}{\mathrm{~d} \mathcal{E}^{2}}-\left(\frac{1}{r} \frac{\mathrm{d} r}{\mathrm{~d} \mathcal{E}}+\frac{1}{2 a} \frac{\mathrm{d} a}{\mathrm{~d} \mathcal{E}}\right) \frac{\mathrm{d} x}{\mathrm{~d} \mathcal{E}}\right] .
\end{aligned}
$$

We set $x=r$ in Eq. (22) and use the result in Eq. (19). After multiplying both sides by $r^{2} a$, we get:

$$
\frac{\mathrm{d}^{2} r}{\mathrm{~d}^{2}}-\frac{1}{r}\left(\frac{\mathrm{d} r}{\mathrm{~d} \mathcal{E}}\right)^{2}-\frac{h^{2} a}{r}+a=\left(\mathbf{f}_{p} \cdot \mathbf{i}\right) r^{2} a+\frac{1}{2 a} \frac{\mathrm{d} a}{\mathrm{~d} \mathcal{E}} \frac{\mathrm{d} r}{\mathrm{~d} \mathcal{E}}
$$

Since the term containing the derivative of $a$ vanishes in the case of pure Kepler motion, it is of the character of a perturbing term and it is consequently shifted to the right-hand side. From the expression of the non-dimensional Kepler energy:

$$
U_{\mathrm{K}}=\frac{1}{2 r}\left[\frac{1}{r a}\left(\frac{\mathrm{d} r}{\mathrm{~d} \mathcal{E}}\right)^{2}+\frac{h^{2}}{r}-2\right]
$$

after rearranging the terms, we obtain:

$$
\frac{1}{r a}\left(\frac{\mathrm{d} r}{\mathrm{~d} \mathcal{E}}\right)^{2}+\frac{h^{2}}{r}=2\left(r U_{\mathrm{K}}+1\right) .
$$

Both sides are then multiplied by $-a$, and $U_{\mathrm{K}}$ is substituted by $-1 /(2 a)$. Some simplifications lead to the equality:

$$
-\frac{1}{r}\left(\frac{\mathrm{d} r}{\mathrm{~d} \mathcal{E}}\right)^{2}-\frac{h^{2} a}{r}=r-2 a .
$$

The previous relation is straight applied to Eq. (23), which becomes:

$$
\frac{\mathrm{d}^{2} r}{\mathrm{~d} \mathcal{E}^{2}}+r-a=\left(\mathbf{f}_{p} \cdot \mathbf{i}\right) r^{2} a+\frac{1}{2 a} \frac{\mathrm{d} a}{\mathrm{~d} \mathcal{E}} \frac{\mathrm{d} r}{\mathrm{~d} \mathcal{E}}
$$

Note that this equation is not singular when $r=0$ : the original Eq. (19) has been regularized.

What we are going to do now is to use the variation of parameters technique in order to find the set of two regular elements attached to $r$ and $\mathrm{d} r / \mathrm{d} \mathcal{E}$, and the differential equations which describe their behavior. In the pure Kepler motion the terms on the right-hand side of Eq. (24) disappear to give:

$$
\frac{\mathrm{d}^{2} r}{\mathrm{~d} \mathcal{E}^{2}}+r=a
$$

This equation can be analytically integrated and the solution is represented by:

$$
r=a+A \cos \mathcal{E}+B \sin \mathcal{E},
$$

where $A$ and $B$ are integration constants which depend on the initial conditions. In Eq. (25) we prefer to collect $a$, and introduce the elements $\eta_{1}$ and $\eta_{2}$ :

$$
r=a\left(1-\eta_{1} \cos \mathcal{E}-\eta_{2} \sin \mathcal{E}\right)
$$

From the comparison of Eq. (26) with the well-known expression of the orbital radius (here non-dimensional) in terms of the eccentric anomaly $E$ :

$$
r=a(1-e \cos E)
$$

and by comparing the two expressions for the radial velocity:

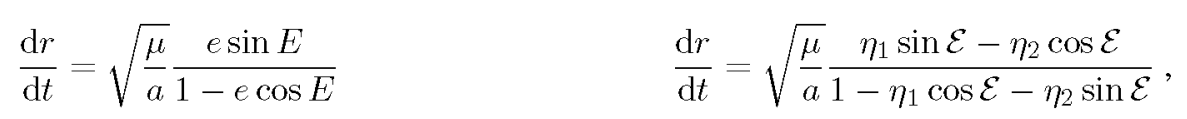

we infer the following relations for $\eta_{1}$ and $\eta_{2}$ :

$$
\begin{aligned}
& \eta_{1}=e \cos (\mathcal{E}-E) \\
& \eta_{2}=e \sin (\mathcal{E}-E) .
\end{aligned}
$$


We substitute Eq. (24) with the corresponding system of two first-order differential equations:

$$
\begin{aligned}
& \frac{\mathrm{d} r}{\mathrm{~d} \mathcal{E}}=w \\
& \frac{\mathrm{d} w}{\mathrm{~d} \mathcal{E}}+r-a=\left(\mathbf{f}_{p} \cdot \mathbf{i}\right) r^{2} a+\frac{w}{2 a} \frac{\mathrm{d} a}{\mathrm{~d} \mathcal{E}} .
\end{aligned}
$$

A solution of the perturbed problem is sought in the form:

$$
\begin{aligned}
& r=\eta_{3}(\mathcal{E})\left[1-\eta_{1}(\mathcal{E}) \cos \mathcal{E}-\eta_{2}(\mathcal{E}) \sin \mathcal{E}\right] \\
& w=\eta_{3}(\mathcal{E})\left[\eta_{1}(\mathcal{E}) \sin \mathcal{E}-\eta_{2}(\mathcal{E}) \cos \mathcal{E}\right]
\end{aligned}
$$

where, from now on, $a$ is replaced by $\eta_{3}$. If these relations are introduced in Eqs. (29) and (30), the differential equations governing the evolution of $\eta_{1}$ and $\eta_{2}$ are obtained. They are reported below together with the derivative of $\eta_{3}$ with respect to $\mathcal{E}$ :

$$
\begin{aligned}
\frac{\mathrm{d} \eta_{1}}{\mathrm{~d} \mathcal{E}} & =\eta_{3}^{2}\left\{\left(l^{2} \sin \mathcal{E}-2 \hat{r} \eta_{2}\right) f_{p x}+l\left[(1+\hat{r}) \cos \mathcal{E}-\eta_{1}\right] f_{p y}\right\} \\
\frac{\mathrm{d} \eta_{2}}{\mathrm{~d} \mathcal{E}} & =\eta_{3}^{2}\left\{\left(-l^{2} \cos \mathcal{E}+2 \hat{r} \eta_{1}\right) f_{p x}+l\left[(1+\hat{r}) \sin \mathcal{E}-\eta_{2}\right] f_{p y}\right\} \\
\frac{\mathrm{d} \eta_{3}}{\mathrm{~d} \mathcal{E}} & =2 \eta_{3}^{3}\left[\left(\eta_{1} \sin \mathcal{E}-\eta_{2} \cos \mathcal{E}\right) f_{p x}+l f_{p y}\right],
\end{aligned}
$$

where the quantities $\hat{r}$ and $l$ are defined by:

$$
\begin{aligned}
& \hat{r}=\frac{r}{a}=1-\eta_{1} \cos \mathcal{E}-\eta_{2} \sin \mathcal{E} \\
& l=\sqrt{1-e^{2}}=\sqrt{1-\eta_{1}^{2}-\eta_{2}^{2}} .
\end{aligned}
$$

Finally, we report the time equation (20) in terms of $\eta_{1}, \eta_{2}$ and $\eta_{3}$ :

$$
\frac{\mathrm{d} \tau}{\mathrm{d} \mathcal{E}}=\eta_{3}^{3 / 2} \hat{r},
$$

where $\hat{r}$ is provided by Eq. (31).

Equations (27) and (28) define $\eta_{1}$ and $\eta_{2}$ as the projections of the eccentricity vector on two orthogonal axes which lie on the osculating orbital plane. Because $\eta_{1}, \eta_{2}$ and $\mathrm{e}$ are integrals of the motion, we infer that the two axes are fixed with respect to inertial space as long as the motion is unperturbed, as we are going to show.

Let us introduce the orbital frame $\mathcal{P}=\langle\mathbf{m}, \mathbf{n}, \mathbf{k}\rangle$ defined as follows: the axis $\mathbf{k}$ is oriented as the osculating angular momentum vector, the axis $\mathbf{m}$ lies on the osculating plane and is rotated counter-clockwise around $\mathbf{k}$ by the angle $E$ (eccentric anomaly) with respect to the eccentricity vector e, and the axis $\mathbf{n}$ completes the setup to yield a Cartesian dextral system. The angular velocity of $\mathcal{P}$ is given by:

$$
\boldsymbol{\omega}_{E}=\frac{r}{h} f_{p z} \mathbf{i}+\left[\frac{h}{r^{2}}-\frac{\mathrm{d}(\vartheta-E)}{\mathrm{d} \tau}\right] \mathbf{k},
$$

where $\mathbf{i}$ is the unit position vector. With the help of the differential equations of $E$ and $\vartheta$ derived with Poisson's variational method, which are respectively given by Eq. (21) and the equation:

$$
\frac{\mathrm{d} \vartheta}{\mathrm{d} \tau}=\frac{h}{r^{2}}+\frac{\partial \vartheta}{\partial \mathbf{v}} \mathbf{f}_{p}
$$

the component of $\omega_{E}$ along $\mathbf{k}$ is written in the form:

$$
\omega_{E z}=\omega_{E} \cdot \mathbf{k}=\frac{\mathrm{d} \mathcal{E}}{\mathrm{d} \tau}-\frac{\partial(\vartheta-E)}{\partial \mathbf{v}} \cdot \mathbf{f}_{p} .
$$

So, as we expected, when the perturbing acceleration is zero $\left(f_{p}=0\right), \mathcal{P}$ rotates at the angular velocity of $d \mathcal{E} / d \tau$ around the fixed axis $\mathbf{k}$.

Let $\mathcal{S}=\left\langle\mathbf{s}_{1}, \mathbf{s}_{2}, \mathbf{s}_{3}\right\rangle$ be a reference frame that is defined by the rotation:

$$
\left[\mathbf{s}_{1}, \mathbf{s}_{2}, \mathbf{s}_{3}\right]=[\mathbf{m}, \mathbf{n}, \mathbf{k}] Q,
$$


where $Q$ is the matrix:

$$
Q=\left(\begin{array}{ccc}
\cos \mathcal{E} & \sin \mathcal{E} & 0 \\
-\sin \mathcal{E} & \cos \mathcal{E} & 0 \\
0 & 0 & 1
\end{array}\right)
$$

The eccentricity vector is projected on $\mathcal{S}$, to yield:

$$
\mathbf{e}=e \cos (\mathcal{E}-E) \mathbf{s}_{1}+e \sin (\mathcal{E}-E) \mathbf{s}_{2},
$$

and by substituting Eqs. (27) and (28) in the equation above, we get:

$$
\mathbf{e}=\eta_{1} \mathbf{s}_{1}+\eta_{2} \mathbf{s}_{2}
$$

The angular velocity of $\mathcal{S}$ relative to $\mathcal{P}$ is:

$$
\boldsymbol{\Omega}_{E, r e l}=-\frac{\mathrm{d} \mathcal{E}}{\mathrm{d} \tau} \mathbf{k}
$$

so that the angular velocity of $\mathcal{S}$ becomes:

$$
\boldsymbol{\Omega}_{E}=\frac{r}{h} f_{p z} \mathbf{i}-\left[\frac{\partial(\vartheta-E)}{\partial \mathbf{v}} \cdot \mathbf{f}_{p}\right] \mathbf{k}
$$

We conclude our proof by stating that the elements $\eta_{1}$ and $\eta_{2}$ are the projections of $\mathbf{e}$ on the axes $\mathbf{s}_{1}$ and $\mathbf{s}_{2}$ of the reference frame $\mathcal{S}$, as shown by Eq. (38), and that $\mathcal{S}$, according to Eq. (39), is inertially fixed when the motion is unperturbed.

\subsubsection{Introducing a time-element}

We introduce an element with respect to the physical time $\tau$, called the time-element. In order to derive it we consider the case of pure Kepler motion. By integrating Eq. (33), the variable time is obtained:

$$
\tau=C_{0}+\eta_{3}^{3 / 2} \mathcal{E}-\eta_{3}^{3 / 2}\left(\eta_{1} \sin \mathcal{E}-\eta_{2} \cos \mathcal{E}\right)
$$

where $C_{0}$ is the constant of integration. The term:

$$
\tau^{*}=C_{0}+\eta_{3}^{3 / 2} \mathcal{E}
$$

which linearly depends on the independent variable $\mathcal{E}$, is the time-element. Let us, firstly, plug Eq. (41) into Eq. (40) and rearrange the terms to get:

$$
\tau^{*}=\tau+\eta_{3}^{3 / 2}\left(\eta_{1} \sin \mathcal{E}-\eta_{2} \cos \mathcal{E}\right)
$$

Then, we differentiate Eq. (42) with respect to $\mathcal{E}$. After exploiting Eq. (33) and simplifying, it results:

$$
\frac{\mathrm{d} \tau^{*}}{\mathrm{~d} \mathcal{E}}=\sqrt{\eta_{3}}\left[\eta_{3}\left(1+\frac{\mathrm{d} \eta_{1}}{\mathrm{~d} \mathcal{E}} \sin \mathcal{E}-\frac{\mathrm{d} \eta_{2}}{\mathrm{~d} \mathcal{E}} \cos \mathcal{E}\right)+\frac{3}{2} \frac{\mathrm{d} \eta_{3}}{\mathrm{~d} \mathcal{E}}\left(\eta_{1} \sin \mathcal{E}-\eta_{2} \cos \mathcal{E}\right)\right]
$$

Once $\tau^{*}$ is known, the physical time is calculated by the relation:

$$
\tau=\tau^{*}-\eta_{3}^{3 / 2}\left(\eta_{1} \sin \mathcal{E}-\eta_{2} \cos \mathcal{E}\right)
$$

\subsection{The unit quaternion}

We present two possible formulations for the unit quaternion.

\subsubsection{Reference frame $\mathcal{R}$}

Let us consider the reference frame $\mathcal{R}$, which was defined in (1). The evolution of the unit quaternion $\mathbf{p}=$ $\left(p_{1}, p_{2}, p_{3}\right), p_{4}$ associated to $\mathcal{R}$ in the independent variable $\mathcal{E}$, is governed by the relations:

$$
\frac{\mathrm{d} \mathbf{p}}{\mathrm{d} \mathcal{E}}=\frac{r \sqrt{a}}{2}\left(\mathbf{p} \times \boldsymbol{\omega}+p_{4} \boldsymbol{\omega}\right) \quad \frac{\mathrm{d} p_{4}}{\mathrm{~d} \mathcal{E}}=-\frac{r \sqrt{a}}{2} \mathbf{p} \cdot \boldsymbol{\omega}
$$


where we remember that all the quantities involved are non-dimensional, and $\omega$ is the angular velocity vector of $\mathcal{R}$. After substituting the expression of $\omega$, given by Eq. (3), the four derivatives take the form:

$$
\begin{aligned}
\frac{\mathrm{d} p_{1}}{\mathrm{~d} \mathcal{E}} & =\frac{\sqrt{a}}{2}\left(\frac{h}{r} p_{2}+\frac{r^{2} f_{p z}}{h} p_{4}\right) \\
\frac{\mathrm{d} p_{2}}{\mathrm{~d} \mathcal{E}} & =-\frac{\sqrt{a}}{2}\left(\frac{h}{r} p_{1}-\frac{r^{2} f_{p z}}{h} p_{3}\right) \\
\frac{\mathrm{d} p_{3}}{\mathrm{~d} \mathcal{E}} & =\frac{\sqrt{a}}{2}\left(\frac{h}{r} p_{4}-\frac{r^{2} f_{p z}}{h} p_{2}\right) \\
\frac{\mathrm{d} p_{4}}{\mathrm{~d} \mathcal{E}} & =-\frac{\sqrt{a}}{2}\left(\frac{h}{r} p_{3}+\frac{r^{2} f_{p z}}{h} p_{1}\right) .
\end{aligned}
$$

The differential equations of the elements attached to the components of the unit quaternion $p=\left(p_{1}, p_{2}, p_{3}, p_{4}\right)$ are determined by applying the variation of parameters technique to Eqs. (45) - (48) (see Peláez et al. (2007)). A direct way of obtaining these equations is to multiply both sides of Eqs. (13) - (16) by:

$$
\frac{\mathrm{d} \sigma}{\mathrm{d} \mathcal{E}}=\frac{h \sqrt{a}}{r} .
$$

We have:

$$
\begin{aligned}
\frac{\mathrm{d} p_{10}}{\mathrm{~d} \mathcal{E}} & =G f_{p z}\left[\cos \left(\sigma-\sigma_{0}\right) p_{40}-\sin \left(\sigma-\sigma_{0}\right) p_{30}\right] \\
\frac{\mathrm{d} p_{20}}{\mathrm{~d} \mathcal{E}} & =G f_{p z}\left[\cos \left(\sigma-\sigma_{0}\right) p_{30}+\sin \left(\sigma-\sigma_{0}\right) p_{40}\right] \\
\frac{\mathrm{d} p_{30}}{\mathrm{~d} \mathcal{E}} & =-G f_{p z}\left[\cos \left(\sigma-\sigma_{0}\right) p_{20}-\sin \left(\sigma-\sigma_{0}\right) p_{10}\right] \\
\frac{\mathrm{d} p_{40}}{\mathrm{~d} \mathcal{E}} & =-G f_{p z}\left[\cos \left(\sigma-\sigma_{0}\right) p_{10}+\sin \left(\sigma-\sigma_{0}\right) p_{20}\right]
\end{aligned}
$$

where $G$ is the product of $g$, defined by Eq. (18), with $\mathrm{d} \sigma / \mathrm{d} \mathcal{E}$. The quantity $G$ can be explicitated in terms of $\eta_{1}$, $\eta_{2}, \eta_{3}$ and $\mathcal{E}$ as follows:

$$
G=\frac{\eta_{3}^{2}\left(1-\eta_{1} \cos \mathcal{E}-\eta_{2} \sin \mathcal{E}\right)^{2}}{2 \sqrt{1-\eta_{1}^{2}-\eta_{2}^{2}}}
$$

Because $\sigma$ appears in Eqs. (50) - (53), it is added to the set of integration variables and its derivative with respect to $\mathcal{E}$ is written in a suitable form for being integrated:

$$
\frac{\mathrm{d} \sigma}{\mathrm{d} \mathcal{E}}=\frac{\sqrt{1-\eta_{1}^{2}-\eta_{2}^{2}}}{1-\eta_{1} \cos \mathcal{E}-\eta_{2} \sin \mathcal{E}} .
$$

In the pure Kepler problem Eq. (54) is analytically integrable by separation of variables to yield:

$$
\sigma=2 \tan ^{-1} \frac{\left(1+\eta_{1}\right) \tan \left(\frac{\mathcal{\varepsilon}}{2}\right)-\eta_{2}}{\sqrt{1-\eta_{1}^{2}-\eta_{2}^{2}}}+C_{0},
$$

where $C_{0}$ is the constant of integration.

\subsubsection{Reference frame $\mathcal{P}$}

Let $\mathbf{u s ~ c o n s i d e r ~ t h e ~ r e f e r e n c e ~ f r a m e ~} \mathcal{P}=\langle\mathbf{m}, \mathbf{n}, \mathbf{k}\rangle$, previously introduced. The axes $\mathbf{m}$ and $\mathbf{n}$ of $\mathcal{P}$ may be defined as:

$$
\begin{aligned}
& \mathbf{m}=\cos (\vartheta-E) \mathbf{i}-\sin (\vartheta-E) \mathbf{j} \\
& \mathbf{n}=\sin (\vartheta-E) \mathbf{i}+\cos (\vartheta-E) \mathbf{j},
\end{aligned}
$$

where $\mathbf{i}, \mathbf{j}$ and $\mathbf{k}$ are the unit vectors of the reference frame $\mathcal{R}$ provided in (1). Let $\mathbf{b}=\left(b_{1}, b_{2}, b_{3}\right), b_{4}$ be the unit quaternion associated to $\mathcal{P}$. Then, we exploit the relations (44), wherein $\boldsymbol{\omega}$ is replaced by $\boldsymbol{\omega}_{E}$, given in Eq. (34), to 
get:

$$
\begin{aligned}
& \frac{\mathrm{d} b_{1}}{\mathrm{~d} \mathcal{E}}=\frac{\sqrt{a}}{2}\left\{r \omega_{E z} b_{2}+\frac{r^{2} f_{p z}}{h}\left[\cos (\vartheta-E) b_{4}-\sin (\vartheta-E) b_{3}\right]\right\} \\
& \frac{\mathrm{d} b_{2}}{\mathrm{~d} \mathcal{E}}=\frac{\sqrt{a}}{2}\left\{-r \omega_{E z} b_{1}+\frac{r^{2} f_{p z}}{h}\left[\cos (\vartheta-E) b_{3}+\sin (\vartheta-E) b_{4}\right]\right\} \\
& \frac{\mathrm{d} b_{3}}{\mathrm{~d} \mathcal{E}}=\frac{\sqrt{a}}{2}\left\{r \omega_{E z} b_{4}-\frac{r^{2} f_{p z}}{h}\left[\cos (\vartheta-E) b_{2}-\sin (\vartheta-E) b_{1}\right]\right\} \\
& \frac{\mathrm{d} b_{4}}{\mathrm{~d} \mathcal{E}}=-\frac{\sqrt{a}}{2}\left\{r \omega_{E z} b_{3}+\frac{r^{2} f_{p z}}{h}\left[\cos (\vartheta-E) b_{1}+\sin (\vartheta-E) b_{2}\right]\right\},
\end{aligned}
$$

where $\omega_{E z}$ is reported in Eq. (36). When the motion is unperturbed Eqs. (58) - (61) simplify in:

$$
\begin{aligned}
\frac{\mathrm{d} b_{1}}{\mathrm{~d} \mathcal{E}} & =\frac{1}{2} b_{2} \\
\frac{\mathrm{d} b_{2}}{\mathrm{~d} \mathcal{E}} & =-\frac{1}{2} b_{1} \\
\frac{\mathrm{d} b_{3}}{\mathrm{~d} \mathcal{E}} & =\frac{1}{2} b_{4} \\
\frac{\mathrm{d} b_{4}}{\mathrm{~d} \mathcal{E}} & =-\frac{1}{2} b_{3} .
\end{aligned}
$$

This system of four differential equations can be analytically integrated to provide the solutions:

$$
\begin{aligned}
& b_{1}=\cos \left(\frac{\mathcal{E}-\mathcal{E}_{0}}{2}\right) b_{10}+\sin \left(\frac{\mathcal{E}-\mathcal{E}_{0}}{2}\right) b_{20} \\
& b_{2}=\cos \left(\frac{\mathcal{E}-\mathcal{E}_{0}}{2}\right) b_{20}-\sin \left(\frac{\mathcal{E}-\mathcal{E}_{0}}{2}\right) b_{10} \\
& b_{3}=\cos \left(\frac{\mathcal{E}-\mathcal{E}_{0}}{2}\right) b_{30}+\sin \left(\frac{\mathcal{E}-\mathcal{E}_{0}}{2}\right) b_{40} \\
& b_{4}=\cos \left(\frac{\mathcal{E}-\mathcal{E}_{0}}{2}\right) b_{40}-\sin \left(\frac{\mathcal{E}-\mathcal{E}_{0}}{2}\right) b_{30},
\end{aligned}
$$

where $b_{10}, b_{20}, b_{30}$ and $b_{40}$ are constants in the pure Kepler motion, but vary with $\mathcal{E}$ when perturbations are applied. The equations which describe the evolution of these quantities in the perturbed two-body motion are found by plugging Eqs. (62) - (65) into Eqs. (58) - (61). After some algebra we get:

$$
\begin{aligned}
& \frac{\mathrm{d} b_{10}}{\mathrm{~d} \mathcal{E}}=-A \frac{\partial(\vartheta-E)}{\partial \mathbf{v}} \cdot \mathbf{f}_{p} b_{20}+G f_{p z}\left[\cos (\vartheta-E+\triangle \mathcal{E}) b_{40}-\sin (\vartheta-E+\triangle \mathcal{E}) b_{30}\right] \\
& \frac{\mathrm{d} b_{20}}{\mathrm{~d} \mathcal{E}}=A \frac{\partial(\vartheta-E)}{\partial \mathbf{v}} \cdot \mathbf{f}_{p} b_{10}+G f_{p z}\left[\cos (\vartheta-E+\triangle \mathcal{E}) b_{30}+\sin (\vartheta-E+\triangle \mathcal{E}) b_{40}\right] \\
& \frac{\mathrm{d} b_{30}}{\mathrm{~d} \mathcal{E}}=-A \frac{\partial(\vartheta-E)}{\partial \mathbf{v}} \cdot \mathbf{f}_{p} b_{40}-G f_{p z}\left[\cos (\vartheta-E+\triangle \mathcal{E}) b_{20}-\sin (\vartheta-E+\triangle \mathcal{E}) b_{10}\right] \\
& \frac{\mathrm{d} b_{40}}{\mathrm{~d} \mathcal{E}}=A \frac{\partial(\vartheta-E)}{\partial \mathbf{v}} \cdot \mathbf{f}_{p} b_{30}-G f_{p z}\left[\cos (\vartheta-E+\triangle \mathcal{E}) b_{10}+\sin (\vartheta-E+\triangle \mathcal{E}) b_{20}\right],
\end{aligned}
$$

where $\triangle \mathcal{E}=\mathcal{E}-\mathcal{E}_{0}$ and:

$$
A=\frac{\eta_{3}^{3 / 2}}{2}\left(1-\eta_{1} \cos \mathcal{E}-\eta_{2} \sin \mathcal{E}\right) .
$$

The trigonometric functions in Eqs. (66) - (69) can be expressed in terms of $\eta_{1}, \eta_{2}$ and $\mathcal{E}$ by means of the identities:

$$
\begin{aligned}
& e \cos E=\eta_{1} \cos \mathcal{E}+\eta_{2} \sin \mathcal{E} \\
& e \sin E=\eta_{1} \sin \mathcal{E}-\eta_{2} \cos \mathcal{E} .
\end{aligned}
$$


If in place of $\vartheta-E$ in Eqs. (56) and (57) we set $\sigma-\mathcal{E}$, Eqs. (66) - (69) take a more simple form:

$$
\begin{aligned}
\frac{\mathrm{d} \tilde{b}_{10}}{\mathrm{~d} \mathcal{E}} & =G f_{p z}\left[\cos \left(\sigma-\mathcal{E}_{0}\right) \tilde{b}_{40}-\sin \left(\sigma-\mathcal{E}_{0}\right) \tilde{b}_{30}\right] \\
\frac{\mathrm{d} \tilde{b}_{20}}{\mathrm{~d} \mathcal{E}} & =G f_{p z}\left[\cos \left(\sigma-\mathcal{E}_{0}\right) \tilde{b}_{30}+\sin \left(\sigma-\mathcal{E}_{0}\right) \tilde{b}_{40}\right] \\
\frac{\mathrm{d} \tilde{b}_{30}}{\mathrm{~d} \mathcal{E}} & =-G f_{p z}\left[\cos \left(\sigma-\mathcal{E}_{0}\right) \tilde{b}_{20}-\sin \left(\sigma-\mathcal{E}_{0}\right) \tilde{b}_{10}\right] \\
\frac{\mathrm{d} \tilde{b}_{40}}{\mathrm{~d} \mathcal{E}} & =-G f_{p z}\left[\cos \left(\sigma-\mathcal{E}_{0}\right) \tilde{b}_{10}+\sin \left(\sigma-\mathcal{E}_{0}\right) \tilde{b}_{20}\right],
\end{aligned}
$$

where $\sigma$ is calculated by integrating Eq. (54).

\subsection{Set of differential equations}

We collect below the differential equations of the seven generalized orbital elements $\left(\eta_{1}, \eta_{2}, \eta_{3}, p_{10}, p_{20}, p_{30}, p_{40}\right)$ :

$$
\begin{aligned}
& \frac{\mathrm{d} \eta_{1}}{\mathrm{~d} \mathcal{E}}=\eta_{3}^{2}\left\{\left(l^{2} \sin \mathcal{E}-2 \hat{r} \eta_{2}\right) f_{p x}+l\left[(1+\hat{r}) \cos \mathcal{E}-\eta_{1}\right] f_{p y}\right\} \\
& \frac{\mathrm{d} \eta_{2}}{\mathrm{~d} \mathcal{E}}=\eta_{3}^{2}\left\{\left(-l^{2} \cos \mathcal{E}+2 \hat{r} \eta_{1}\right) f_{p x}+l\left[(1+\hat{r}) \sin \mathcal{E}-\eta_{2}\right] f_{p y}\right\} \\
& \frac{\mathrm{d} \eta_{3}}{\mathrm{~d} \mathcal{E}}=2 \eta_{3}^{3}\left[\left(\eta_{1} \sin \mathcal{E}-\eta_{2} \cos \mathcal{E}\right) f_{p x}+l f_{p y}\right] \\
& \frac{\mathrm{d} p_{10}}{\mathrm{~d} \mathcal{E}}=G f_{p z}\left[\cos \left(\sigma-\sigma_{0}\right) p_{40}-\sin \left(\sigma-\sigma_{0}\right) p_{30}\right] \\
& \frac{\mathrm{d} p_{20}}{\mathrm{~d} \mathcal{E}}=G f_{p z}\left[\cos \left(\sigma-\sigma_{0}\right) p_{30}+\sin \left(\sigma-\sigma_{0}\right) p_{40}\right] \\
& \frac{\mathrm{d} p_{30}}{\mathrm{~d} \mathcal{E}}=-G f_{p z}\left[\cos \left(\sigma-\sigma_{0}\right) p_{20}-\sin \left(\sigma-\sigma_{0}\right) p_{10}\right] \\
& \frac{\mathrm{d} p_{40}}{\mathrm{~d} \mathcal{E}}=-G f_{p z}\left[\cos \left(\sigma-\sigma_{0}\right) p_{10}+\sin \left(\sigma-\sigma_{0}\right) p_{20}\right]
\end{aligned}
$$

where:

$$
\begin{aligned}
& \hat{r}=\frac{r}{a}=1-\eta_{1} \cos \mathcal{E}-\eta_{2} \sin \mathcal{E} \\
& l=\sqrt{1-e^{2}}=\sqrt{1-\eta_{1}^{2}-\eta_{2}^{2}} \\
& G=\frac{\left(\eta_{3} \hat{r}\right)^{2}}{2 l} .
\end{aligned}
$$

We complete the set of Eqs. (74) - (80) with the equations of the variable $\sigma$ and the physical time $\tau$ :

$$
\begin{aligned}
& \frac{\mathrm{d} \sigma}{\mathrm{d} \mathcal{E}}=\frac{l}{\hat{r}}, \\
& \frac{\mathrm{d} \tau}{\mathrm{d} \mathcal{E}}=\eta_{3}^{3 / 2} \hat{r} .
\end{aligned}
$$

The time-element $\tau^{*}$ might be implemented in place of $\tau$ :

$$
\frac{\mathrm{d} \tau^{*}}{\mathrm{~d} \mathcal{E}}=\sqrt{\eta_{3}}\left[\eta_{3}\left(1+\frac{\mathrm{d} \eta_{1}}{\mathrm{~d} \mathcal{E}} \sin \mathcal{E}-\frac{\mathrm{d} \eta_{2}}{\mathrm{~d} \mathcal{E}} \cos \mathcal{E}\right)+\frac{3}{2} \frac{\mathrm{d} \eta_{3}}{\mathrm{~d} \mathcal{E}}\left(\eta_{1} \sin \mathcal{E}-\eta_{2} \cos \mathcal{E}\right)\right]
$$

\subsection{Peláez's formulation with a different independent variable}

We change in Eqs. (10) - (16) the independent variable from $\sigma$ to $\widehat{\mathcal{E}}$, according to the relation:

$$
\sigma=2 \tan ^{-1}\left(\sqrt{\frac{1+e}{1-e}} \tan \frac{\widehat{\mathcal{E}}}{2}\right),
$$

where $e$ is the eccentricity. Both hands of the previous equation are differentiated with respect to $\sigma$ to find out the quantity:

$$
\frac{\mathrm{d} \widehat{\mathcal{E}}}{\mathrm{d} \sigma}=\frac{1-e \cos \hat{\mathcal{E}}}{\sqrt{1-e^{2}}}-\frac{\sin \widehat{\mathcal{E}}}{1-e^{2}} \frac{\mathrm{d} e}{\mathrm{~d} \sigma},
$$


Table 1. Comparison of special perturbation methods for the problem of oblate Earth plus the Moon

\begin{tabular}{lccc}
\hline & Steps/Revolution & RSS $(\mathrm{km})$ & $\triangle R(\mathrm{~km})$ \\
\hline Stiefel-Scheifele & 62 & 0.0144 & 0.0005 \\
DROMO & 62 & 0.0973 & 0.0973 \\
DROMO-B & 62 & 0.1228 & 0.1060 \\
DROMO-A & 62 & 47.6757 & 0.6035 \\
DROMO-A-te & 62 & 46.2256 & 0.6138 \\
Cowell & 240 & 45.2104 & 24.5627 \\
\hline
\end{tabular}

where:

$$
\frac{\mathrm{d} e}{\mathrm{~d} \sigma}=\frac{1}{\sqrt{\zeta_{1}^{2}+\zeta_{2}^{2}}}\left(\zeta_{1} \frac{\mathrm{d} \zeta_{1}}{\mathrm{~d} \sigma}+\zeta_{2} \frac{\mathrm{d} \zeta_{2}}{\mathrm{~d} \sigma}\right) .
$$

The set of elements is the same of Peláez's method and is reported in Eq. (9). The corresponding differential equations in the new independent variable $\widehat{\mathcal{E}}$ are obtained by dividing Eqs. (10) - (16) by $\mathrm{d} \hat{\mathcal{E}} / \mathrm{d} \sigma$ given in Eq. (82). Finally, the following identities, which are consequences of Eq. (81), are employed:

$$
\begin{aligned}
\cos \sigma & =\frac{\cos \widehat{\mathcal{E}}-e}{1-e \cos \widehat{\mathcal{E}}} \\
\sin \sigma & =\frac{\sqrt{1-e^{2}} \sin \widehat{\mathcal{E}}}{1-e \cos \widehat{\mathcal{E}}} .
\end{aligned}
$$

\section{Results}

We present numerical comparisons of the following schemes:

1. The original formulation of Peláez's method (see Peláez et al. (2007)). Let us name it DROMO.

2. The two modified versions of Pelaez's method, with and without the time-element, whose equations are reported in section 3.3. Let us name them DROMO-A and DROMO-A-te respectively.

3. The modified version of Pelaez's method presented in section 3.4. Let us call it DROMO-B.

4. Stiefel and Scheifele's set of regular elements linked to the Kustaanheimo-Stiefel parameters (see Stiefel and Scheifele (1971) for a review).

5. Cowell's method.

Two problems are used for the comparisons: the first deals with a satellite in an highly eccentric orbit perturbed by (1) the Earth oblateness and (2) the Lunar perturbation; the second problem deals with a continuous radial thrust applied to a satellite in an initial circular orbit. In order to integrate the differential equations, the Runge-Kutta method of fourth order with Cash-Karp parameters was applied.

\subsection{Oblate Earth plus the Moon}

The problem, which is the example $2 \mathrm{~b}$ of the book by Stiefel and Scheifele (1971) (page 122) is to determine the position of the satellite after 50 revolutions $(288.12768941 \mathrm{msd})$ with the initial position and velocity vectors expressed in an inertial reference frame by:

$$
\begin{aligned}
& \left(x_{1}, x_{2}, x_{3}\right)=(0.0,-5888.9727,-3400.0) \mathrm{km} \\
& \left(\dot{x}_{1}, \dot{x}_{2}, \dot{x}_{3}\right)=(10.691338,0.0,0.0) \mathrm{km} \mathrm{s}^{-1} .
\end{aligned}
$$

The unperturbed Kepler orbit has an inclination of $30^{\circ}$ with respect to the equator $\left(x_{1}, x_{2}\right.$-plane) and an eccentricity of 0.95 . The satellite is initially at the pericenter at distance $R=6800 \mathrm{~km}$. Details on the implementation of the two perturbations are available in the book Stiefel and Scheifele (1971). The solution regarded as correct was given in Stiefel and Scheifele (1971):

$$
\left(x_{1, \text { ref }}, x_{2, \text { ref }}, x_{3, \text { ref }}\right)=(-24219.0503,227962.1064,129753.4424) \mathrm{km} \text {, }
$$



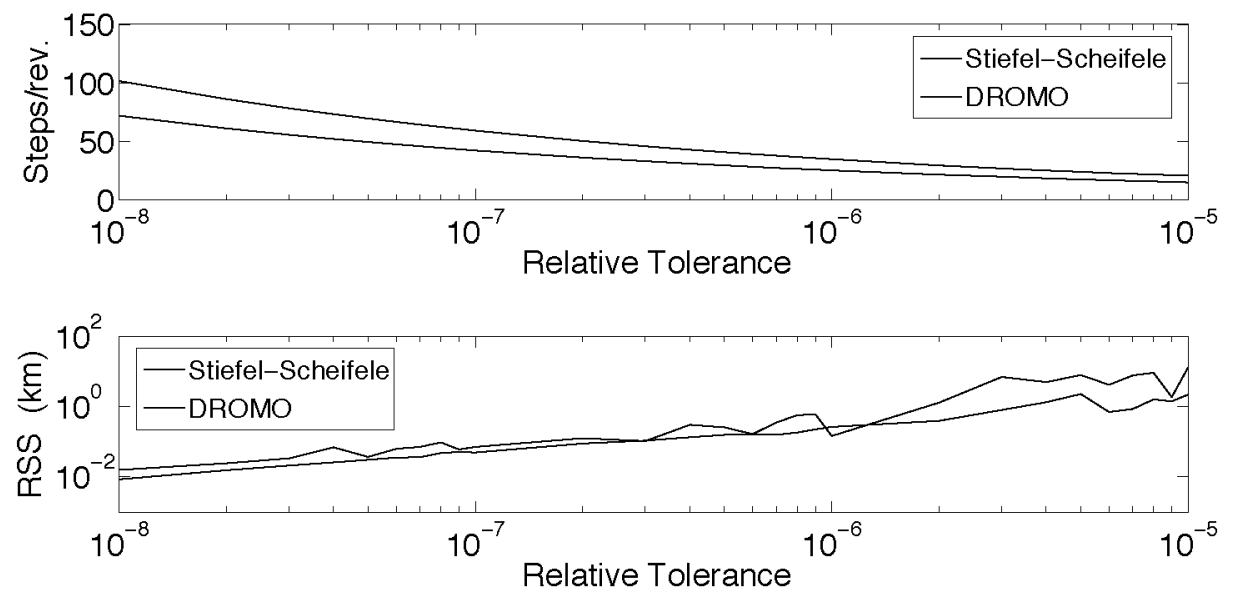

Figure 2. Steps per revolution and RSS of the error in function of the relative tolerance of the RK 4(5) for Stiefel-Scheifele's method and DROMO

and was obtained by integrating the differential equations of Stiefel-Scheifele's method with 498 steps per revolution.

Bond and Allman in their book (Bond and Allman, 1996) use this problem in order to compare different methods. The solution (83) was assumed as exact and the error calculated as the distance between the final position vector provided by each method and the exact one. Also Peláez exploits the problem to test the performance of his special perturbation method (Peláez et al., 2007), which proves to be the most accurate among the other methods compared: Sperling-Burdet, Kustaanheimo-Stiefel and Cowell.

We compare our selected methods by imposing a frequency of 62 steps of integration per revolution and recording two different errors. The first is calculated as in the reference Bond and Allman (1996):

$$
\mathrm{RSS}=\sqrt{\left(x_{1, \mathrm{f}}-x_{1, \text { ref }}\right)^{2}+\left(x_{2, \mathrm{f}}-x_{2, \text { ref }}\right)^{2}+\left(x_{3, \mathrm{f}}-x_{3, \text { ref }}\right)^{2}},
$$

where $\left(x_{1, \mathrm{f}}, x_{2, \mathrm{f}}, x_{3, \mathrm{f}}\right)$ is the final position obtained from a generic method. The second is the difference between the magnitudes of the position vectors:

$$
\triangle R=\left|\sqrt{x_{1, \mathrm{f}}^{2}+x_{2, \mathrm{f}}^{2}+x_{3, \mathrm{f}}^{2}}-\sqrt{x_{1, \text { ref }}^{2}+x_{2, \text { ref }}^{2}+x_{3, \text { ref }}^{2}}\right| .
$$

The results are shown in Tab. (1). The best performance belongs to Stiefel-Scheifele's method in both the value of RSS and that of $\triangle R$. Second ranked is DROMO, which shows a slightly better accuracy of DROMO-B. The RSS of Peláez's special perturbation method is about $97 \mathrm{~m}$, which is appreciably smaller than the value $250 \mathrm{~m}$ obtained by Peláez (Peláez et al., 2007). Note that DROMO-A and DROMO-A-te have a RSS higher than $46 \mathrm{~km}$, and a $\triangle R$ smaller than $1 \mathrm{~km}$ : this means that the error is mainly in the orientation of the position vector, while its magnitude is propagated with a good accuracy. Finally, Cowell's method is the least accurate, even if we aware that we are not using an appropriate numerical integrator for this formulation.

Figure (2) compares the first two ranked methods, Stiefel-Scheifele and DROMO, in terms of steps per revolution and RSS, which are plotted in function of the relative tolerance of the Runge-Kutta algorithm.

\subsection{Continuous radial thrust}

A satellite is orbiting around the Earth in an circular orbit of radius $r_{0}=6800 \mathrm{~km}$. At time $t=0$, it is propelled by a constant radial thrust of:

$$
a_{p x}=1.22719913916381 \times 10^{-3} \frac{\mathrm{km}}{\mathrm{s}^{2}} .
$$

The problem is to determine the magnitude of the position vector of the satellite after $t_{\mathrm{f}}=10000 \mathrm{~s}$. The solution can be analytically calculated, thanks to Tsien's procedure (see Battin (1999)), and results:

$$
R_{\text {ref }}=22735.0635 \mathrm{~km} .
$$


Table 2. Comparison of special perturbation methods for the problem of continuous radial thrust

\begin{tabular}{lccc}
\hline & Steps & $R_{\mathrm{f}}(\mathrm{km})$ & $\triangle R(\mathrm{~km})$ \\
\hline Solution & - & 22735.0635 & - \\
DROMO-B & 200 & 22735.0635 & $1.17 \times 10^{-5}$ \\
DROMO-A & 200 & 22735.0636 & $3.54 \times 10^{-5}$ \\
DROMO-A-te & 200 & 22735.0636 & $3.54 \times 10^{-5}$ \\
Stiefel-Scheifele & 200 & 22735.0636 & $8.00 \times 10^{-5}$ \\
DROMO & 200 & 22735.0636 & $8.39 \times 10^{-5}$ \\
Cowell & 200 & 22734.6704 & 0.39 \\
\hline
\end{tabular}

Also this problem is reported in the book by Bond and Allman (1996), but they set a final time of $t_{\mathrm{f}}=12000 \mathrm{~s}$, which corresponds to escape from the Earth. The selected methods are compared with a required number of 200 steps of integration per revolution, and the error is calculated as the difference with respect to the reference distance. The results are reported in Tab. (2). The modifications of Peláez's special perturbation method proposed in this paper are the most accurate in predicting the final distance of the satellite, and so they beat Stiefel-Scheifele's method, whose performance is similar to that of DROMO.

\section{Conclusions}

Starting from the special perturbation method developed by Peláez (Peláez et al., 2007), new formulations for the case of elliptic motion have been developed. The three new methods are named DROMO-A, DROMO-A-te and DROMO-B. The comparison of these schemes with the original version of Pelaez's method (named DROMO) and also with the very efficient Stiefel-Scheifele's method is shown by using the problems of oblate Earth plus Moon and continuous radial thrust. In the first problem Stiefel-Scheifele's method is the most accurate, followed by DROMO, which slightly improves the very good accuracy of DROMO-B. In the second problem all the new versions of Peláez's method developed in this paper show a better performance with respect to Stiefel-Scheifele's method.

\section{References}

Arakida, H. and Fukushima, T. Long-term integration error of Kustaanheimo-Stiefel regularized orbital motion. II. Method of variation of parameters. The Astronomical Journal, 121:1764-1767, March 2001.

Battin, R. H. An Introduction to the Mathematics and Methods of Astrodynamics. AIAA Education Series, AIAA, Reston, VA, revised edition, 1999.

Boccaletti, D. and Pucacco, G. Theory of Orbits, volume 1. Springer, Berlin, 1999.

Bond, V. R. and Allman, M. C. Modern Astrodynamics: Fundamentals and Perturbation Methods. Princeton University Press, Princeton, NJ, 1996.

Deprit, A., Elipe, A., and Ferrer, S. Linearization: Laplace vs. Stiefel. Celestial Mechanics and Dynamical Astronomy, 58:151-201, 1994.

Fukushima, T. Efficient integration of highly eccentric orbits by scaling methods applied to Kustaanheimo-Stiefel regularization. The Astronomical Journal, 128:3114-3122, December 2004.

Peláez, J., Hedo, J. M., and de Andréz, P. R. A special perturbation method in orbital dynamics. Celestial Mechanics and Dynamical Astronomy, 97(2):131-150, 2007.

Stiefel, L. and Scheifele, G. Linear and Regular Celestial Mechanics. Springer-Verlag, New York Heidelberg Berlin, 1971. 